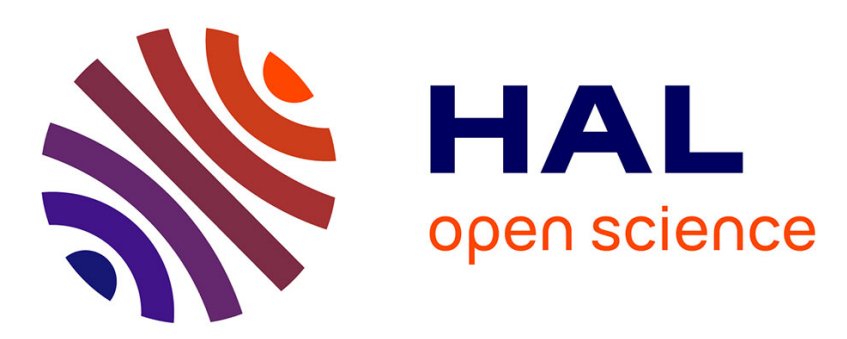

\title{
Tensor-Based Receiver With Adaptive Relay Activation for Two-Hop Cooperative Systems
}

Antonio Augusto Teixeira Peixoto, Pedro Marinho R. de Oliveira

\section{To cite this version:}

Antonio Augusto Teixeira Peixoto, Pedro Marinho R. de Oliveira. Tensor-Based Receiver With Adaptive Relay Activation for Two-Hop Cooperative Systems. XXXVI Simpósio Brasileiro de Telecomunicações e Processamento de Sinais (SBRT 2018), Sep 2018, Campina Grande, Brazil. 10.14209/sbrt.2018.208. hal-01954335

\section{HAL Id: hal-01954335 https://hal.science/hal-01954335}

Submitted on 13 Dec 2018

HAL is a multi-disciplinary open access archive for the deposit and dissemination of scientific research documents, whether they are published or not. The documents may come from teaching and research institutions in France or abroad, or from public or private research centers.
L'archive ouverte pluridisciplinaire HAL, est destinée au dépôt et à la diffusion de documents scientifiques de niveau recherche, publiés ou non, émanant des établissements d'enseignement et de recherche français ou étrangers, des laboratoires publics ou privés. 


\title{
Tensor-Based Receiver With Adaptive Relay Activation for Two-Hop Cooperative Systems
}

\author{
Antonio Augusto Teixeira Peixoto and Pedro Marinho R. de Oliveira
}

\begin{abstract}
In this paper, it is proposed a relay activation method for a multiuser cooperative uplink system, based on the current Signal-to-Noise Ratio (SNR) of the link between relay and base station. Depending on this current SNR, extra relays can be activated, enhancing the quality of the received signal and making the uplink transmission less susceptible to unpredictable SNR variations. The communication system is modeled as a PARAllel FACtor (PARAFAC) tensor decomposition, exploiting its uniqueness properties to estimate the transmitted symbols, channel gains and spatial signatures of the users. The proposed method is based on the iterative algorithm Alternating Least Squares (ALS). Since the receiver can estimate the channel gains, a real-time change of the number of relays would not deteriorate the channel's coefficients estimations. Computer simulations based on Monte Carlo runs show the performance of the proposed relay activation method.
\end{abstract}

Keywords-Cooperative Communications, PARAFAC, Tensor Decompositions, Relay Activation, Alternating Least Squares.

\section{INTRODUCTION}

Significant gains can be achieved by means of cooperative diversity [1], such as an increase of the coverage area, less path losses, uncorrelated channels, higher throughput and an overall better link quality [2]. This process exploits the cooperation between nodes of the same network to achieve better transmission quality. In cooperative communication models, paths with uncorrelated fading between the destination and the source node are generated through the introduction of one or more retransmission devices called relays [1,2]. This technique has emerged as a promising wireless access solution to extend coverage without using high power levels at the transmitter, hence increasing connectivity and capacity [3].

Moreover, the use of tensor decompositions has been gaining attention in several signal processing applications [4,5]. For wireless communication systems, especially in situations where the received signal can be viewed as multidimensional variables [6,7], the use of high-order arrays allows a better understanding and processing in a multidimensional perspective. Hence, the practical motivation for a tensor modeling comes from the fact that we can exploit simultaneously multiple forms of diversity to perform multiuser signal separation, equalization and channel estimation under uniqueness conditions more relaxed than matrix decompositions.

The work [6] pioneered the use of tensor decompositions in telecommunications by proposing a semi-blind receiver

Antonio Augusto Teixeira Peixoto is with the Federal University of Ceará, CE, Brazil. (email: augusto.peixoto@outlook.com). Pedro Marinho R. de Oliveira is with the Université Côte d'Azur, I3S Laboratory, CNRS, France. (e-mail: marinho@i3s.unice.fr). based on a PARAllel FACtor (PARAFAC) [8] decomposition. In [9], another tensor decomposition called PARATUCK was adopted for a wireless Multiple-Input Multiple-Output (MIMO) system. Also, tensor analysis has shown to be an efficient approach for channel and symbol estimation in cooperative communications, as the works $[10,11,12,13]$. In [10], a semi-blind receiver was proposed for a cooperative Amplify-and-Forward (AF) relaying scenario with a trilinear tensor adopted and transmissions of the relays in different time slots. [11] generalized the work of [10] for different relaying schemes. In [12] a cooperative uplink with simultaneous relay transmission and spreading at the relays was considered. [13] generalized the works of [10] and [11] by adopting a quadrilinear PARAFAC decomposition and proposing a semiblind Alternating Least Squares (ALS)-based receiver. We can cite works on cooperative MIMO systems, as in [14], where two iterative semi-blind receivers were formulated for a twohop MIMO AF relaying system. In [15], a Nested-PARAFAC tensor model is proposed and two iterative semi-blind receivers are proposed, jointly estimating the symbol and the channels of the communication links. In [16], the PARATUCK3 decomposition is used to model the communication of a three-hop MIMO cooperative system. In this work, two noniterative semi-blind receivers are proposed to jointly estimate the symbols and channels matrices.

In this paper, it is proposed a relay activation technique for a multiuser cooperative uplink system (based on the models of [10] and [13]), where the received signal is modeled as a PARAFAC tensor decomposition. This is an iterative ALSbased technique that uses the current Signal-to-Noise Ratio (SNR) of the link between relay and base station to activate extra relays, in order to improve the quality of the received signal. The rest of this paper is organized as follows. Section II presents the communication system model, while Section III describes the PARAFAC tensor decomposition. Section IV introduces the proposed algorithm, Section V presents the simulation results and, finally, Section VI ends this paper with the conclusions.

Notation: Scalars are denoted by Roman lower-case letters $(a, b, \ldots)$, vectors as lower-case boldface letters $(\mathbf{a}, \mathbf{b}, \ldots)$, matrices as upper-case boldface letters $(\mathbf{A}, \mathbf{B}, \ldots)$ and tensors as calligraphic letters $(\mathcal{A}, \mathcal{B}, \ldots)$. To retrieve the element $(i, j)$ of an arbitrary matrix $\mathbf{A} \in \mathbb{C}^{I \times J}$, we use $[\mathbf{A}]_{i, j}$ (the same for tensors). $\mathbf{A}^{T}$ and $\mathbf{A}^{\dagger}$ stands for the transpose and the pseudo-inverse of $\mathbf{A}$, respectively. The operator $\operatorname{diag}_{i}[\mathbf{A}]$ is the diagonal matrix formed by the $i^{t h}$ row of $\mathbf{A}$ and $\diamond$ denotes the Khatri-Rao product between $\mathbf{B} \in \mathbb{C}^{I \times R}$ and $\mathbf{C} \in \mathbb{C}^{J \times R}$, resulting in $\mathbf{B} \diamond \mathbf{C} \in \mathbb{C}^{I J \times R}$. 


\section{SYSTEM MODEL}

It is considered a cooperative uplink system with $M$ users, where each user transmits towards a base station with the help of an associated relay link, thus, a two-hop communication system. The relay spreads the user signal in frequency with an individual pseudo-noise (PN) code of length $P$ and forwards the signal. The base station have an array of $K$ antennas, receives the relay signals and estimates the transmitted symbols, channel coefficients and antenna gains of the users.

The users transmits in two phases: the test phase and the adaptation phase. During the test phase, each user have only one active relay. Then, the base station performs the estimations, and, according to the SNR of the relay signals, it can activate extra relays for each user in order to improve the link quality of them. After these estimations, the test phase ends and the adaptation phase begins, where the base station will keep activating extra relays if the SNR of the relay signals drops or it will deactivate if the SNR increases. Users and relays are single antenna devices, both work in semiduplex mode, and the respective links are subject to flat fading. Synchronization at the symbol level was considered for the propagation scenario. We also assume that the users are far distant from each other so interference is not experienced at the relays.

\section{A. Test phase}

At the beginning of the test phase, the user transmits to its associated relay. Figure 1 illustrates the system during the test phase. The signal received by the relay of the $m^{t h}$ user in the user-relay (UR) link is given by:

$$
x_{m, n}^{(U R)}=h_{m}^{(U R)} s_{n, m}+v_{m, n}^{(U R)},
$$

where $h_{m}^{(U R)}$ is the channel coefficient between the $m^{t h}$ user and its relay, $s_{n, m}$ is the $n^{t h}$ symbol of the $m^{\text {th }}$ user, where $n=$ $1, \ldots, N$, and $v_{m, n}^{(U R)}$ is the noise component. The symbols $s_{n, m}$ are all independent and identically distributed over a PhaseShift Keying (PSK) alphabet, with $m=1, \ldots, M$. The relays spread the users signals, and, then, they forward it to the base station by using the AF protocol. The signal received at the $k^{t h}$ antenna of the base station, on the $n^{t h}$ symbol period and $p^{t h}$ chip of the PN sequence, on the relay-base (RB) link of all users, is given by:

$$
x_{k, n, p}^{(R B)}=\sum_{m=1}^{M} h_{k, m}^{(R B)} g_{m} x_{m, n}^{(U R)} c_{p, m}+v_{k, n, p}^{(R B)},
$$

where $h_{k, m}^{(R B)}$ is the channel coefficient between the $k^{t h}$ receiving antenna and the relay associated with the $m^{\text {th }}$ user, $g_{m}$ is the amplification factor applied by the relay of the $m^{t h}$ user, $c_{p, m}$ is the $p^{t h}$ chip of the PN sequence of the $m^{\text {th }}$ user, with $p=1, \ldots, P$, and $v_{k, n, p}^{(R B)}$ is the noise of the RB link. Transmission then occurs in 2 steps. From (1) and (2), we have:

$$
x_{k, n, p}^{(R B)}=\sum_{m=1}^{M} h_{k, m}^{(R B)} h_{m}^{(U R)} g_{m} s_{n, m} c_{p, m}+v_{k, n, p}^{(U R B)},
$$

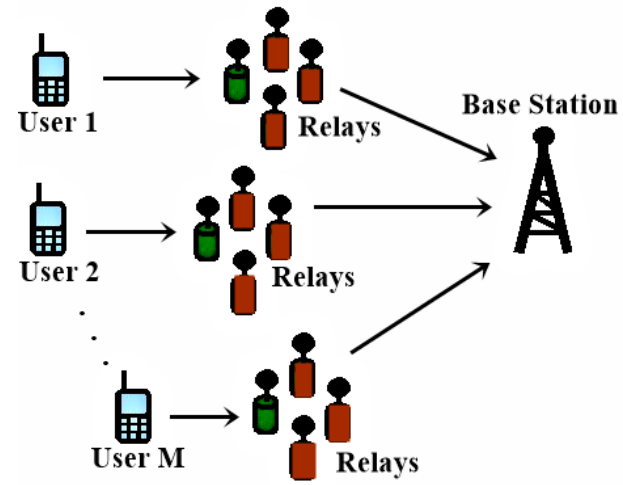

Fig. 1. Cooperative uplink with $M$ users and one active relay (in green) and three deactivated relays (in red) for each user.

$$
v_{k, n, p}^{(U R B)}=\sum_{m=1}^{M} h_{k, m}^{(R B)} g_{m} v_{m, n}^{(U R)} c_{p, m}+v_{k, n, p}^{(R B)} .
$$

with $v_{k, n, p}^{(U R B)}$, we denote the total noise through the user-relaybase (URB) link. Transmission rate during the test phase is given by $M / 2$. After the base station receives the first RB link transmission of all users, the test phase is over.

\section{B. Adaptation phase}

The adaptation phase begins with the base station activating extra relays for all users after receiving the first transmissions. If the SNR of the RB link is below certain thresholds, one or more extra relays are then activated. The extra relays also can be deactivated if the SNR of the RB link surpass those thresholds. Thus, the signal received by the $r^{\text {th }}$ relay of the $m^{t h}$ user is given by:

$$
x_{r, m, n}^{(U R)}=h_{r, m}^{(U R)} s_{n, m}+v_{r, m, n}^{(U R)},
$$

with $h_{r, m}^{(U R)}$ being the channel coefficient between the $m^{\text {th }}$ user and its $r^{\text {th }}$ relay, where $r=1, \ldots, R$, with $R$ being the number of relays currently active for each user and $v_{r, m, n}^{(U R)}$ being the noise component. After receiving the user signal, the relays forwards it in $R$ different time-slots, hence, transmission occurs in $R+1$ steps.

The received signal of the RB links of all users, at the base station, during the adaptation phase, is given by:

$$
x_{k, r, n, p}^{(R B)}=\sum_{m=1}^{M} h_{k, r, m}^{(R B)} g_{r, m} x_{r, m, n}^{(U R)} c_{p, m}+v_{k, r, n, p}^{(R B)},
$$

with $h_{k, r, m}^{(R B)}$ being the channel coefficient between the $k^{t h}$ receiving antenna and the $r^{\text {th }}$ relay associated with the $m^{\text {th }}$ user, $g_{r, m}$ is the amplification factor applied by the $r^{t h}$ relay of the $m^{t h}$ user and $v_{k, r, n, p}^{(R B)}$ is the noise component of the link. Substituting (5) in (6), we have:

$$
\begin{gathered}
x_{k, r, n, p}^{(R B)}=\sum_{m=1}^{M} h_{k, r, m}^{(R B)} h_{r, m}^{(U R)} g_{r, m} s_{n, m} c_{p, m}+v_{k, r, n, p}^{(U R B)}, \\
v_{k, r, n, p}^{(U R B)}=\sum_{m=1}^{M} h_{k, r, m}^{(R B)} g_{r, m} v_{r, m, n}^{(U R)} c_{p, m}+v_{k, r, n, p}^{(R B)} .
\end{gathered}
$$


The noise component of the user-relay-link is given by $v_{k, r, n, p}^{(U R B)}$. Note that during the adaptation phase, the number of relays $R$ can be changed during the transmission periods, so, the size of $x_{k, r, n, p}^{(R B)}$ can vary. Transmission rate during the adaptation phase is given by $M /(R+1)$.

\section{Propagation scenario}

In this subsection we discuss the propagation scenario during the test phase and adaptation phase. Let us consider that all RB links are subject to multipath propagation and the possible scatters are located far away from the base station, then, all the signals transmitted by the relays arrive at the destination with approximately the same angle of arrival. This assumption implies that the angle spread is small compared to the spatial resolution of the antenna array employed at the base station. If the user and its relays are close to each other and there is no scattering around the antennas of the base station, this consideration is valid. In suburban areas, where base stations are usually placed on the top of tall buildings or in towers, this propagation scenario is very common [17]. Hence, during the adaptation phase, the channel coefficient $h_{k, r, m}^{(R B)}$ can be defined as:

$$
h_{k, r, m}^{(R B)}=\sum_{l=1}^{L_{r, m}^{(R B)}} a_{k}\left(\theta_{m}\right) \beta_{l, r, m}^{(R B)}
$$

with $a_{k}\left(\theta_{m}\right)$ being the spatial signature of the $k^{\text {th }}$ base station antenna for the $m^{t h}$ scatter, where $\theta_{m}$ is the mean angle of arrival of the $m^{t h}$ scatter. We define $a_{k}\left(\theta_{m}\right)=\exp \left(j \theta_{m}^{k-1}\right)$, with $k=1, \ldots, K$ and $\theta_{m}$ being an uniform random variable with zero mean and variance of $2 \pi$. The term $\beta_{l, r, m}^{(R B)}$ is the fading envelope of the $l^{t h}$ path between the $r^{t h}$ relay of the $m^{t h}$ user and the base station with $L_{r, m}$ being the total number of multipaths.

Equation (9) can be rewritten as follows:

$$
h_{k, r, m}^{(R B)} \approx a_{k}\left(\theta_{m}\right) \gamma_{r, m}^{(R B)},
$$

with $\gamma_{r, m}^{(R B)}$ being defined as $\gamma_{r, m}^{(R B)}=\sum_{l=1}^{L_{r, m}^{(R B)}} \beta_{l, r, m}^{(R B)}$. Substituting (10) in (7) and in (8), we have:

$$
\begin{aligned}
& x_{k, r, n, p}^{(R B)}=\sum_{m=1}^{M} a_{k}\left(\theta_{m}\right) \gamma_{r, m}^{(R B)} h_{r, m}^{(U R)} g_{r, m} s_{n, m} c_{p, m}+v_{k, r, n, p}^{(U R B)}, \\
& v_{k, r, n, p}^{(U R B)}=\sum_{m=1}^{M} a_{k}\left(\theta_{m}\right) \gamma_{r, m}^{(R B)} g_{r, m} v_{r, m, n}^{(U R)} c_{p, m}+v_{k, r, n, p}^{(R B)} .
\end{aligned}
$$

During the test phase, we have $R=1$, thus $\gamma_{r, m}^{(R B)}$ becomes $\gamma_{1, m}^{(R B)}$ or simply $\gamma_{m}^{(R B)}$.

\section{Relay activation method}

As mentioned in subsection II.B, the base station will activate extra relays if the SNR of the RB link $\left(S N R^{(R B)}\right)$ is below some thresholds. In this paper we have considered that $\Gamma_{1}, \Gamma_{2}$ and $\Gamma_{3}$ are the three possible thresholds for relay activation, where $\Gamma_{1}<\Gamma_{2}<\Gamma_{3}$. The extra relays are activated by control links between the base station and the users associated relays called base-relay (BR) links. The base station will simply broadcast a Binary PSK (BPSK) wave trough the BR links, whereas the user associated relays picks up the BR link signals and activate the corresponding number of extra relays that are nearby. By sending a modulated pulse signal containing $1 \mathrm{~s}$, the number of $1 \mathrm{~s}$ sent defines the number of relays to be activated, and, by sending $0 \mathrm{~s}$, the base station defines the number of relays to be deactivated. These BPSK modulated pulse waves are sent by the base station every time the RB link signals are collected.

It is important to note that the users have no knowledge of how many relays are currently active for them, since they will just transmit to their associated relay and the extra relays will also pick up the signal and forwards it to the base station. Such that, we have the following cases:

- If $S N R^{(R B)}<\Gamma_{1}$, the base station will broadcast three 1s through the BR links and three extra relays are activated for all users.

- If $\Gamma_{1}<S N R^{(R B)}<\Gamma_{2}$, the base station will broadcast two $1 \mathrm{~s}$ and one 0 through the BR links and two extra relays are activated or one relay is deactivated for all users.

- If $\Gamma_{2}<S N R^{(R B)}<\Gamma_{3}$, the base station will broadcast one 1 and two 0 s through the BR links and one extra relay is activated or two relays are deactivated for all users.

- If $\Gamma_{3}<S N R^{(R B)}$, the base station will broadcast three 0s through the BR links and all three extra relays are deactivated for the users.

\section{PARAFAC TENSOR MODEL}

The RB links of both the test and adaptation phase can be viewed as four-way arrays, with its dimensions directly related to space (receiving antennas at the base station), cooperative slots (number of relays), time (symbols) and spreading (chips). In this section, we model the received signals as fourth order tensors, using a PARAFAC decomposition [18]. So, during the test phase, let $\mathcal{X}_{t} \in \mathbb{C}^{K \times 1 \times N \times P}$ be a fourth order tensor collecting the $\mathrm{RB}$ signals at the base station:

$$
\left[\mathcal{X}_{t}\right]_{k, 1, n, p}=x_{k, n, p}^{(R B)}
$$

for $k=1, \ldots, K, n=1, \ldots, N$ and $p=1, \ldots, P$. Omitting the noise terms, we have that an element of $\mathcal{X}_{t}$ is given by:

$$
x_{k, 1, n, p}^{t}=\sum_{m=1}^{M} a_{k}\left(\theta_{m}\right) h_{1, m}^{t} s_{n, m} c_{p, m},
$$

where the term $h_{1, m}^{t}$ is the channel coefficient of the userassociated relay during the test phase and is defined as:

$$
h_{1, m}^{t}=\gamma_{m}^{(R B)} h_{m}^{(U R)} g_{m}
$$

The data tensor shown in (14) corresponds to a PARAFAC decomposition with the following factor matrices: $\mathbf{A} \in \mathbb{C}^{K \times M}$, the antenna array response matrix with $[\mathbf{A}]_{k, m}=a_{k}\left(\theta_{m}\right), \mathbf{H} \in$ $\mathbb{C}^{1 \times M}$, the channel matrix with $[\mathbf{H}]_{1, m}=h_{1, m}^{t}, \mathbf{S} \in \mathbb{C}^{N \times M}$, the symbol matrix with $[\mathbf{S}]_{n, m}=s_{n, m}$ and $\mathbf{C} \in \mathbb{C}^{P \times M}$, the spreading matrix with $[\mathbf{C}]_{p, m}=c_{p, m}$. 
During the adaptation phase, let $\mathcal{X}_{a} \in \mathbb{C}^{K \times R \times N \times P}$ be a fourth order tensor collecting the RB signals at the base station:

$$
\left[\mathcal{X}_{a}\right]_{k, r, n, p}=x_{k, r, n, p}^{(R B)}
$$

for $k=1, \ldots, K, r=1, \ldots, R, n=1, \ldots, N$ and $p=1, \ldots, P$. Again, we omit the AWGN terms, hence, an element of $\mathcal{X}_{a}$ is denoted as:

$$
x_{k, r, n, p}^{a}=\sum_{m=1}^{M} a_{k}\left(\theta_{m}\right) h_{r, m}^{a} s_{n, m} c_{p, m},
$$

with the term $h_{r, m}^{a}$ being the channel coefficient of the users relays during the adaptation phase and it is defined as:

$$
h_{r, m}^{a}=\gamma_{r, m}^{(R B)} h_{r, m}^{(U R)} g_{r, m} .
$$

$\mathcal{X}_{a}$ also corresponds to a PARAFAC tensor with factor matrices: $\mathbf{A}, \mathbf{H} \in \mathbb{C}^{R \times M}$, with $[\mathbf{H}]_{r, m}=h_{r, m}^{a}, \mathbf{S}$ and $\mathbf{C}$. Note that during the adaptation phase the factor matrix $\mathbf{H}$ has dimensions $R \times M$. It is important to mention that a fourth order tensor can be used to model the RB signals of both phases with a minor dimension change representing the addition of extra relays.

\section{A. Matrix unfoldings}

Let $\mathbf{X}_{a}^{(1)} \in \mathbb{C}^{P K R \times N}$ be defined as the tensor $\mathcal{X}_{a} \in$ $\mathbb{C}^{K \times R \times N \times P}$ unfolded into a matrix, during the adaptation phase, as follows:

$$
\mathbf{X}_{a}^{(1)}=(\mathbf{C} \diamond \mathbf{A} \diamond \mathbf{H}) \mathbf{S}^{T},
$$

There are other unfolded matrices, as follows:

$$
\begin{aligned}
& \mathbf{X}_{a}^{(2)}=(\mathbf{S} \diamond \mathbf{C} \diamond \mathbf{A}) \mathbf{H}^{T} \in \mathbb{C}^{N P K \times R}, \\
& \mathbf{X}_{a}^{(3)}=(\mathbf{H} \diamond \mathbf{S} \diamond \mathbf{C}) \mathbf{A}^{T} \in \mathbb{C}^{R N P \times K} .
\end{aligned}
$$

The same matrix unfoldings are used during the test phase, but with $R=1$ (one active relay for each user), thus, for instance, $\mathbf{X}_{t}^{(1)} \in \mathbb{C}^{P K \times N}, \mathbf{X}_{t}^{(2)} \in \mathbb{C}^{N P K \times 1}$ and $\mathbf{X}_{t}^{(3)}$ $\in \mathbb{C}^{N P \times K}$, all obtained the same way as (19)-(21). The PARAFAC decomposition has uniqueness properties that are useful for this system. On certain conditions, the tensor models of (14) and (17) are essentially unique. This uniqueness is granted by Kruskal's condition as showed in [18].

\section{ReCEIVER Algorithm}

The receiver algorithm presented in this section is based on the ALS method, which estimates the factor matrices by using the least squares technique. The basic idea of the ALS procedure in this work is simple: each time, update one of the factor matrices by using the least squares previous estimations of the other factor matrices. Each factor matrix is then estimated in an alternate way, with this procedure being repeated until convergence. The ALS algorithm with adaptive relay activation is shown in Algorithm 1.

Assuming that channel state information is not available at the relays neither at the base station, the receiver uses the unfolding matrices $\mathbf{X}_{t}^{(1)}, \mathbf{X}_{t}^{(2)}, \mathbf{X}_{t}^{(3)}$ and the ones of (19)-(21) to estimate $\mathbf{A}, \mathbf{H}$ and $\mathbf{S}$, where is assumed knowledge of the $\mathrm{PN}$ sequences (matrix $\mathbf{C}$ ) and the first row of $\mathbf{S}$ (one pilot symbol per user) at the receiver.

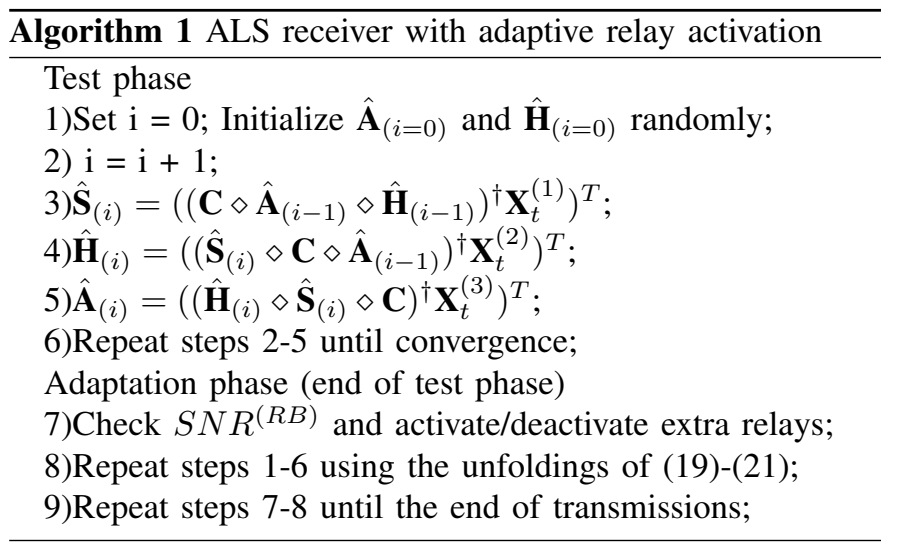

During the test phase, the receiver estimates the factor matrices using $\mathbf{X}_{t}^{(1)}, \mathbf{X}_{t}^{(2)}$ and $\mathbf{X}_{t}^{(3)}$ with the ALS method (steps 1-6 of Algorithm 1), then, after the end of the test phase, the adaptation phase begins and the receiver checks the SNR of the RB link. If $S N R^{(R B)}$ is below or above any of the considered thresholds, the base station sends activation or deactivation signals through the BR links in order to activate or deactivate the corresponding extra relays. When the next transmissions arrive, the receiver repeats the same steps of the ALS method to estimate $\mathbf{A}, \mathbf{H}$ and $\mathbf{S}$ using (19)-(21). This procedure goes on until the end of transmissions. The ALS method achieves convergence when the error between the estimated tensor and the received tensor is below $10^{-6}$.

\section{Simulation Results}

This section presents the results obtained from computer simulations, in order to evaluate the performance of the proposed receiver. The following scenario was adopted. The wireless links have frequency flat Rayleigh fading with path loss exponent equal to 3.5 , the base station antenna array is composed by $K$ antennas, Quadrature PSK (QPSK) modulation is used and PN sequences are considered for spreading. The Symbol Error Rate (SER), Normalized Mean Squared Error (NMSE) and average processing time curves are shown in function of the SNR of the RB link. The mean results were obtained by 20000 independent Monte Carlo runs. The AF relays have variable gains. We set the following parameters: $K=4, P=8, M=4$ and $N=16$. The thresholds for relay activation were set as $\Gamma_{1}=15 \mathrm{~dB}, \Gamma_{2}=27 \mathrm{~dB}$ and $\Gamma_{3}=39$ $\mathrm{dB}$.

Figure 2 shows the SER performance as the SNR increases for the proposed adaptive ALS receiver in comparison to the ones of [10] and [13]. We can see that the proposed adaptive receiver provides a smaller SER for low SNR values than the receivers of [10] and [13] that work with a fixed number of relays $(R=3)$. As the SNR increases, the SER of the adaptive receiver decreases, as the number of relays.

Figure 3 shows the NMSE as the SNR increases of the channels matrix $\mathbf{H}$ of the proposed ALS receiver in comparison to the ones of [10] and [13]. We can see that the performance of the proposed receiver regarding the estimation of the channel matrix $\mathbf{H}$ is similar to the ones compared in this simulation and even better in some SNR intervals. 


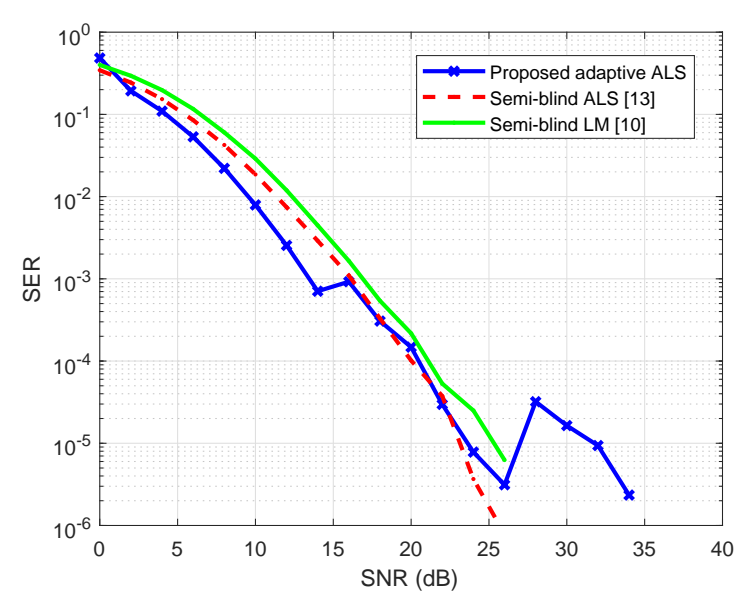

Fig. 2. SER versus SNR - Comparison between different receivers.

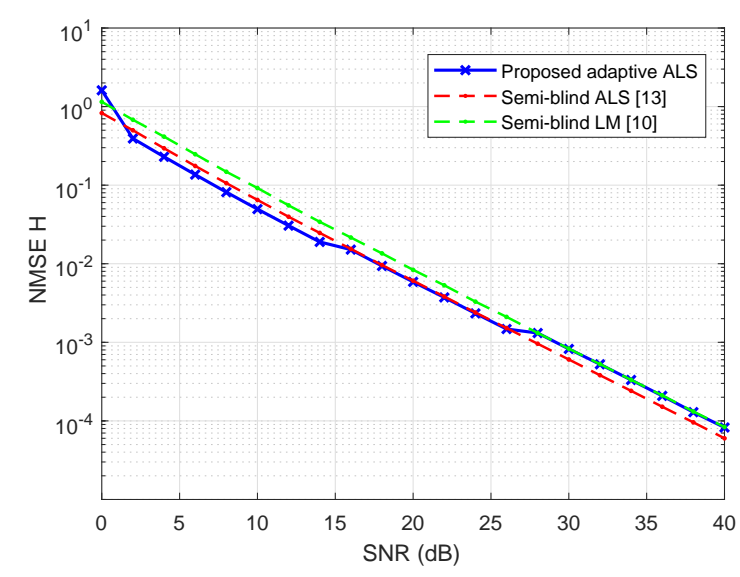

Fig. 3. NMSE versus SNR - Comparison between different receivers.

Finally, Figure 4 shows the average processing time of the proposed ALS receiver in comparison to the one of [13]. A similar average processing time is shown for both receivers.

\section{Conclusions}

In this paper, we have proposed a tensor-based receiver that can jointly and semi-blindly estimate the channel gains, antenna array responses and transmitted symbols of the system. The estimation consists in using the ALS method. After each estimation, the base station can activate or deactivate extra relays for all users, based on the SNR of the RB link. The proposed receiver provided a satisfactory performance regarding the estimation of the symbols and the channels gains, compared to others receivers in the state of the art. In future works, we aim to generalize the proposed receiver for the multi-hop scenario, that is, where more than three relays are used to assist the communication system.

\section{REFERENCES}

[1] Sendonaris, A., Erkip, E., \& Aazhang, B. (2003). User cooperation diversity. Part I. System description. IEEE Transactions on communications, 51(11), 1927-1938.

[2] Sendonaris, A., Erkip, E., \& Aazhang, B. (2003). User cooperation diversity-Part II: Implementation aspects and performance analysis. IEEE Trans. Commun, 51(11), 1939-1948.

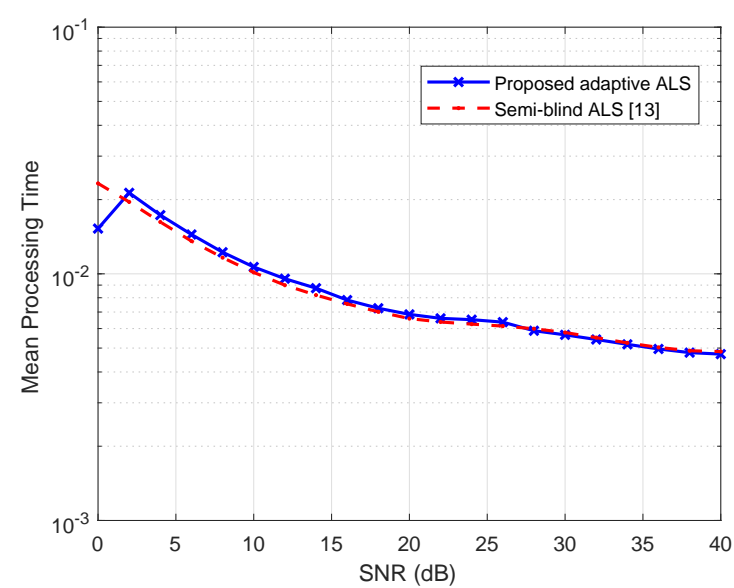

Fig. 4. Average processing time versus SNR - Comparison.

[3] Laneman, J. N., Tse, D. N., \& Wornell, G. W. (2004). Cooperative diversity in wireless networks: Efficient protocols and outage behavior. IEEE Transactions on Information theory, 50(12), 3062-3080.

[4] Kolda, T. G., \& Bader, B. W. (2009). Tensor decompositions and applications. SIAM review, 51(3), 455-500.

[5] Cichocki, A., Mandic, D., De Lathauwer, L., Zhou, G., Zhao, Q., Caiafa C., \& Phan, H. A. (2015). Tensor decompositions for signal processing applications: From two-way to multiway component analysis. IEEE Signal Processing Magazine, 32(2), 145-163.

[6] Sidiropoulos, N. D., Giannakis, G. B., \& Bro, R. (2000). Blind PARAFAC receivers for DS-CDMA systems. IEEE Transactions on Signal Processing, 48(3), 810-823.

[7] Sidiropoulos, N. D., Bro, R., \& Giannakis, G. B. (2000). Parallel factor analysis in sensor array processing. IEEE transactions on Signal Processing, 48(8), 2377-2388.

[8] Bro, R. (1997). PARAFAC. Tutorial and applications. Chemometrics and intelligent laboratory systems, 38(2), 149-171.

[9] Favier, G., Da Costa, M. N., De Almeida, A. L., \& Romano, J. M. T. (2012). Tensor space-time (TST) coding for MIMO wireless communication systems. Signal Processing, 92(4), 1079-1092.

[10] Fernandes, A. R., de Almeida, A. L., \& da Costa, D. B. (2011, June). Blind receiver for amplify-and-forward cooperative diversity scheme. In Signal Processing Advances in Wireless Communications (SPAWC), 2011 IEEE 12th International Workshop on (pp. 546-550). IEEE.

[11] Fernandes, C. A. R., de Almeida, A. L. F., \& da Costa, D. B. (2012). Unified tensor modeling for blind receivers in multiuser uplink cooperative systems. IEEE Signal Processing Letters, 19(5), 247-250.

[12] de Almeida, A. L., Fernandes, C. A., \& da Costa, D. B. (2013). Multiuser detection for uplink DS-CDMA amplify-and-forward relaying systems. IEEE Signal Processing Letters, 20(7), 697-700.

[13] Peixoto, A. A. T., \& Fernandes, C. A. R. (2017). Tensor-Based Multiuser Detection for Uplink DS-CDMA Systems with Cooperative Diversity. XXXV Simpósio Brasileiro de Telecomunicações e Processamento de Sinais (SBrT 2017), (São Pedro, Brazil).

[14] Ximenes, L. R., Favier, G., de Almeida, A. L., \& Silva, Y. C. (2014). PARAFAC-PARATUCK semi-blind receivers for two-hop cooperative MIMO relay systems. IEEE Trans. Signal Process, 62(14), 3604-3615

[15] Ximenes, L. R., Favier, G., \& de Almeida, A. L. (2015). Semi-blind receivers for non-regenerative cooperative MIMO communications based on nested PARAFAC modeling. IEEE transactions on signal processing, 63(18), 4985-4998

[16] P. M. R. de Oliveira \& Fernandes, C. A. R. (2017). PARATUCK-3 SemiBlind Receivers for Three-Hop Cooperative MIMO Relay Systems. XXXV Simpósio Brasileiro de Telecomunicações e Processamento de Sinais (SBrT 2017), (São Pedro, Brazil).

[17] Ertel, R. B., Cardieri, P., Sowerby, K. W., Rappaport, T. S., \& Reed, J. H. (1998). Overview of spatial channel models for antenna array communication systems. IEEE Personal Communications, 5(1), 10-22.

[18] Stegeman, A., \& Sidiropoulos, N. D. (2007). On Kruskal's uniqueness condition for the Candecomp/Parafac decomposition. Linear Algebra and its applications, 420(2-3), 540-552. 